Annals of Pure and Applied Mathematics

Vol. 15, No. 2, 2017, 277-288

ISSN: 2279-087X (P), 2279-0888(online)

Published on 11 December 2017

www.researchmathsci.org

DOI: http://dx.doi.org/10.22457/apam.v15n2a14

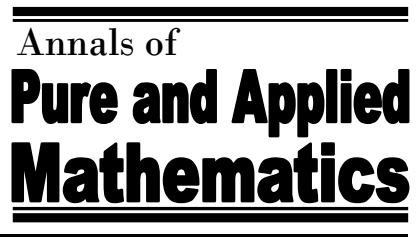

\title{
Magnetohydrodynamic Casson Fluid Flow with Hall Effect and Rotation in a Porous Medium
}

\author{
P.Thirunavukarasu ${ }^{1}$ and S.Bhuvaneswari ${ }^{2}$ \\ ${ }^{1}$ P. G. and Research Department of Mathematics \\ Periyar E.V.R. College (Autonomous), Tiruchirappalli-620 023, India \\ ${ }^{2}$ P. G. and Research Department of Mathematics \\ Kamban College Of Arts And Science for Women, Tiruvannamalai, India
}

Received 25 November2017; accepted 07 December 2017

\begin{abstract}
This paper presents the study of magnetohydrodynamic flow version of the Casson fluid flow with Hall effect and Rotation in the presence of an inclined magnetic field and porous medium. Exact solution of the governing equation is obtained by Similarity transform technique for the MHD flow of incompressible, electrically conducting, Casson fluid past a uniformly accelerated and insulated infinite plate. The effects of the Hall parameter, Hartmann number, Angle of inclination, Porosity parameter and the Rotation parameter on the velocity components are shown graphically. We concluded that the axial velocity decrease and transverse velocity increase for increasing values of Magnetic parameter and Porosity parameter but it is converse in the case of Hall parameter. Both the velocity decreases when the values of Casson fluid parameter and Rotation parameter are increased.
\end{abstract}

Keywords: MHD flow, Casson fluid, uniformly accelerated plate, Hall effect, Porous medium

\section{AMS Mathematics Subject Classification (2010): 76SXX}

\section{Introduction}

The influence of magnetic field over rotation has drawn considerable attention for so many years because it is fundamental to various geophysical phenomena, such as geomagnetic dynamo, solar spin down etc. The steady and unsteady Ekman layers of incompressible fluid have been investigated as basic boundary layers in a rotating fluid appears in the oceanic, atmospheric, cosmic fluid dynamics and solar physics or geophysical problems.

Gupta (1972) analyzed the effect of a uniform transverse magnetic field on Ekman layer. The hydromagnetic boundary layer and hydromagnetic multiple boundary layers in a rotating fluid was investigated by Debnath (1972, 1975). Guchhait et al (2012) investigated the combined effect of Hall current and rotation on MHD flow in a rotaing vertical channel.

In the last two decades, voluminous research has been performed on flow through porous media. The applications of porous media are seen in various fields such 


\section{P.Thirunavukarasu and S.Bhuvaneswari}

as hydrology, environmental pollution, insulation of building and equipment, energy storage and recovery, geothermal reservoirs, nuclear waste disposal and the storage of heat generating materials such as coal.

A porous medium or a porous material is a solid (often called frame or matrix) permeated by an interconnected network of pores (voids) filled with a fluid (liquid or gas). The concept of porous media is used in many areas of applied science and engineering mechanics (acoustics, geomechanics, soil mechanics, rock mechanics), engineering (hydrology, petroleum geology, geophysics), biology and biophysics, material science etc.

Casson fluid is defined as a shear thinning liquid which is understood to have an immeasurable viscosity at zero rate of shear, a yield stress below which no flow occurs and a zero viscosity at an infinite rate of shear Dash (1996). A variety of experiments executed on blood with varying anti-coagulants, temperatures, haematocrits and strongly recommend the performance of blood the same as a Casson fluid (Blair, 1959 and Charm, 1965). Due to a immense application and motivated in this area, an attempt is made to study the effect of Coriolis force and magnetohydrodynamics in Casson fluid flow with Hall current and porous medium.

\section{Formulation of the problem}

Consider the flow of an incompressible electrically conducting, Casson fluid past an infinite and insulated porous flat plate occupying the plane $y=0$. Initially the fluid and the plate rotate in unison with an uniform angular velocity $\bar{\Omega}$ about the y - axis normal to the plate. The $x$-axis is taken in the direction of the motion of the plate and $z$-axis lying on the plate normal to both $x$ and $\mathrm{y}$ - axis. Relative to the rotating fluid, the plate is impulsively started from rest and set into motion with uniform acceleration in its own plane along the $x$ - axis. An uniform magnetic field $H_{0}$, parallel to $y$ - axis is imposed and the plate is electrically non conducting.

The Physical configuration and the nature of the flow suggest the following form of velocity vector $\bar{q}$, magnetic induction vector $\bar{H}$, the uniform angular velocity $\bar{\Omega}$, the Casson fluid parameter $\gamma, k$ is the permeability, electro static field $\bar{E}$ and Pressure $P$, thus $\bar{q}=(u, 0, w), \bar{H}=\left(H_{x}, H_{0}, H_{z}\right), \bar{\Omega}=\left(0, \Omega_{y}, 0\right), \bar{E}=\left(E_{x}, 0, E_{z}\right)$ and $P$ is Constant. The rheological equation of state for an isotropic and incompressible flow of a Casson fluid is as follows [8]

$$
\tau_{i j}=\left\{\begin{array}{cc}
2\left(\mu_{B}+\frac{P_{y}}{\sqrt{2 \pi}}\right) e_{i j}, & \pi>\pi_{c} \\
2\left(\mu_{B}+\frac{P_{y}}{\sqrt{2 \pi_{c}}}\right) e_{i j}, & \pi<\pi_{c}
\end{array}\right.
$$

where $\pi=e_{i j} e_{i j}$ and $e_{i j}$ are the $(i, j) t h$ component of the deformation rate, $\pi$ is the product of the component of deformation rate with itself, $\pi_{c}$ is a critical value of this product based on the non-Newtonian model, $\mu_{B}$ is plastic dynamic viscosity of the nonNewtonian fluid and $P_{y}$ is the yield stress of the fluid. The basic equations with reference to the rotating frame governing the unsteady flow in the presence of magnetic field and Hall current are as follows:

$\nabla \cdot \bar{q}=0$ 
Magnetohydrodynamic Casson Fluid Flow With Hall Effect and Rotation in a Porous Medium

$\frac{\partial \bar{q}}{\partial t}+(\bar{q} \cdot \nabla) \overline{\mathrm{q}}+2 \bar{\Omega} \times \overline{\mathrm{q}}=-\frac{1}{\rho} \nabla \mathrm{P}+v\left(1+\frac{1}{\gamma}\right) \nabla^{2} \overline{\mathrm{q}}+\frac{1}{\rho}(\overline{\mathrm{J}} \times \overline{\mathrm{B}})-\frac{v}{k} \overline{\mathrm{q}}$

The generalized Ohm's law, neglecting ion-slip effect but taking Hall current into account is,

$\frac{\overline{\mathrm{J}}}{\sigma}=(\bar{E}+\overline{\mathrm{q}} \times \bar{B})-\frac{\bar{j} \times \bar{B}}{n \cdot e}$

where $\sigma=\frac{e^{2} m}{m_{e}}$ (is the electrical conductivity).

The initial and boundary conditions are

$u=0, w=0$ for all $t \leq 0$ and for all $y$

$u=U_{0}, w=0$ for all $t>0$ and $y=0$,

$u \rightarrow 0, w \rightarrow 0$ for all $t>0$ and $y \rightarrow \infty$

Physical quantities are cast in non-dimensional form by using the following nondimensional scheme.

$y^{*}=\frac{U_{0} y}{v}, \quad u^{*}=\frac{u}{U_{0}}, \quad w^{*}=\frac{w}{U_{0}}, \quad t^{*}=\frac{U_{0}^{2} t}{v}$

Now introducing the above non-dimensional quantities in equation (2), the components are

$\frac{\partial u}{\partial t}=\left(1+\frac{1}{\gamma}\right) \frac{\partial^{2} u}{\partial y^{2}}-\frac{\sigma H_{0}^{2} v}{\rho U_{0}^{2}\left(1+\omega^{2} \tau^{2}\right)}(u+\omega \tau w)-\frac{2 v}{U_{0}^{2}} w \Omega_{y}-\frac{v^{2} u}{k U_{0}^{2}}$

$\frac{\partial w}{\partial t}=\left(1+\frac{1}{\gamma}\right) \frac{\partial^{2} w}{\partial y^{2}}-\frac{\sigma H_{0}^{2} v}{\rho U_{0}^{2}\left(1+\omega^{2} \tau^{2}\right)}(\omega \tau u-w)+\frac{2 v}{U_{0}^{2}} u \Omega_{y}-\frac{v^{2} w}{k U_{0}^{2}}$

where the non-dimensional parameters are defined below

$M^{2}=\frac{\sigma H_{0}^{2} v}{\rho U_{0}^{2}}$ is the square of the Hartmann number,

$m=\omega \tau$ is the Hall Parameter

$K^{2}=\frac{\nu \Omega_{y}}{U_{0}^{2}}$ is the Rotation parameter i.e., the reciprocal of Ekmann number and

$K_{p}=\frac{v^{2}}{k U_{0}^{2}}$ is the Porosity parameter.

The corresponding initial and boundary conditions (4) in non-dimensional forms are $t \leq 0: u(y, t)=0 ; w(y, t)=0$ for all $y$.

$t>0: u(0, t)=1, w(0, t)=0$

$t>0: u(y, t) \rightarrow 0, w(y, t) \rightarrow 0$ as $y \rightarrow \infty$

\section{Solution of the problem}

By introducing $q=u+i w$, equation (6) and (7) becomes

$\frac{\partial q}{\partial t}=c \frac{\partial^{2} q}{\partial y^{2}}-\left[\left(\frac{M^{2}}{1+m^{2}}\right)(1-i m)+K_{p}-2 i K^{2}\right] q$

where $c=\left(1+\frac{1}{\gamma}\right)$

The initial and boundary conditions take the form

$q(y, 0)=0, q(0, t)=1, \quad q(y, t) \rightarrow 0$ as $y \rightarrow \infty$

Using the abbreviation $\alpha=\left[\left(\frac{M^{2}}{1+m^{2}}\right)(1-i m)+K_{p}-2 i K^{2}\right]$,

Equation (2.9) can be written as

$\frac{\partial q}{\partial t}=c \frac{\partial^{2} q}{\partial y^{2}}-\alpha q$

Also substitute $q(y, t)=e^{i \xi t} g(y)$ in (11), we have 
$c g^{\prime \prime}(y)-(i \xi+\alpha) g(y)=0$

Equation (12) can be solved under the boundary conditions,

$g(0)=e^{-i \xi t}, g(\infty)=0$

The solution is

$g(y)=e^{-i \xi t} e^{\frac{-y}{\sqrt{c}} \sqrt{i \xi+\alpha}}$

Hence $q(y, t)=e^{i \xi t}\left[e^{-i \xi t} e^{-y \sqrt{i \xi+\alpha}}\right]$

Real and imaginary parts of equation (15) are

$u(y, t)=e^{-y S_{1}} \cos y S_{2}$

$w(y, t)=-e^{-y S_{1}} \sin y S_{2}$

where $a=\frac{M^{2}}{1+m^{2}}+K_{p} ; b=-\frac{M^{2} m}{1+m^{2}}-2 K^{2}$;

$S_{1}=\frac{1}{\sqrt{c}} \sqrt{\frac{a+\sqrt{a^{2}+(\xi+b)^{2}}}{2}} ; S_{2}=\frac{1}{\sqrt{c}} \sqrt{\frac{-a+\sqrt{a^{2}+(\xi+b)^{2}}}{2}}$

\section{Shearing stress}

The nondimensional shear stress components $\tau_{x}$ and $\tau_{z}$ at the plate due to the primary and secondary flows are respectively denoted as $\tau_{x}=\left(\frac{\partial u}{\partial y}\right)_{y=0}$ and $\tau_{z}=\left(\frac{\partial w}{\partial y}\right)_{y=0}$ which are derived from equations (16) and (17).

\section{Results and discussion}

Figures 1 and 2 Show the effect of Hartman number in primary and secondary velocity profile. From figure it is clear that, when the Magnetic parameter increases the primary velocity decrease and secondary velocity increases. It is seen from figures 3 and 4 that the primary velocity $u$ increase and secondary velocity $w$ decrease with an increase in Hall parameter. Figures 5,6 and 7,8 depict the primary and secondary velocity profile for increasing values of Casson fluid parameter and rotation parameter $K^{2}$. It is also seen that in both the cases both primary and secondary velocity retards. Figures 9 and 10 displays the velocity profiles for increasing values of porosity parameter and it shows that the primary velocity decrease and secondary velocity increases.

Figure 11-14 shows the primary skin friction $\tau_{x}$ and secondary skin friction $\tau_{z}$ for increasing values of Casson fluid parameter and Porsity parameter respectively. From figures it is clear that primary and secondary shear stress decrease for increasing values of Casson fluid parameter. But the primary shear stress decrease and secondary shear stress increase for increasing values of Porosity parameter.

\section{Conclusion}

- It is concluded that the Primary velocity decrease and secondary velocity increase for increasing values of Magnetic parameter and Porosity parameter but it is converse in the case of Hall parameter.

- The primary and secondary velocity decreases when the values of Casson fluid parameter and Rotation parameter are increased.

- The Shear stress $\tau_{x}$ at the plate decrease with increase of Casson fluid parameter and Porosity parameter. 
Magnetohydrodynamic Casson Fluid Flow With Hall Effect and Rotation in a Porous Medium

- The Shear stress $\tau_{z}$ at the plate decreases when the Casson fluid parameter increased and increases when Porosity parameter is increased with respect to the Hall parameter $m$.

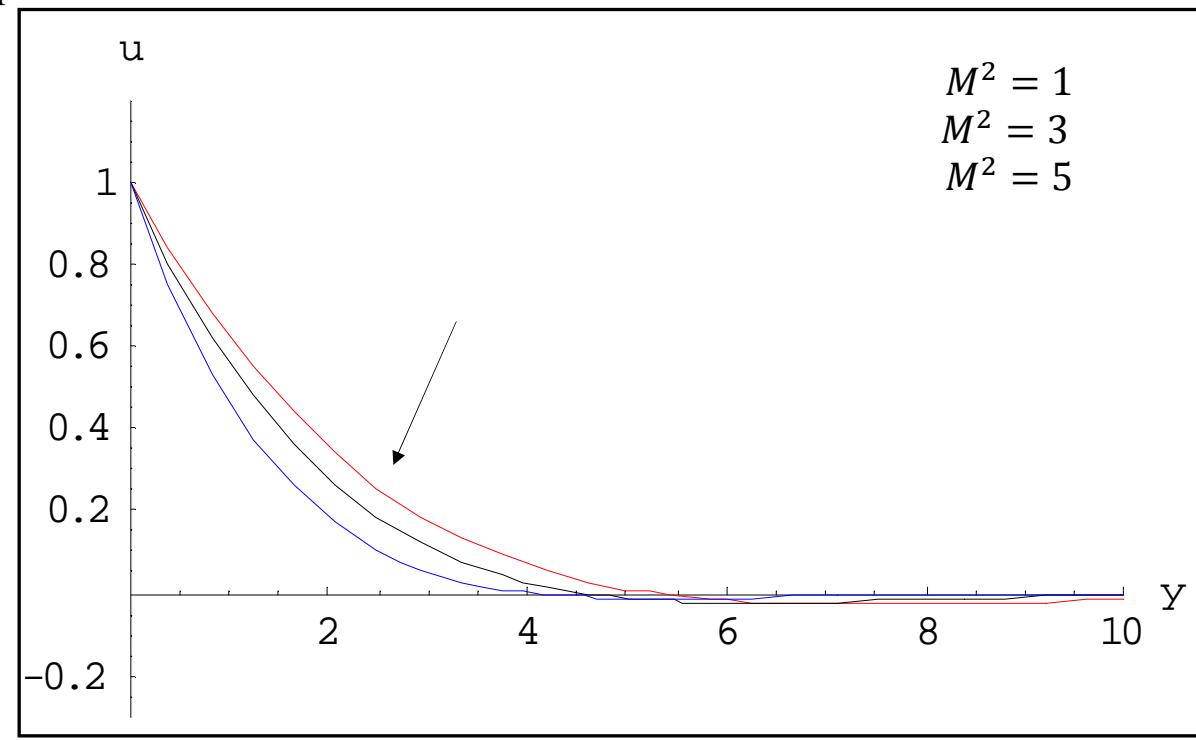

Figure 1: Effect of Hartmann number $\left(M^{2}\right)$ on primary velocity profile when $m=1 ; \xi=1 ; \gamma=0.2 ; K^{2}=1 ; K_{p}=1$

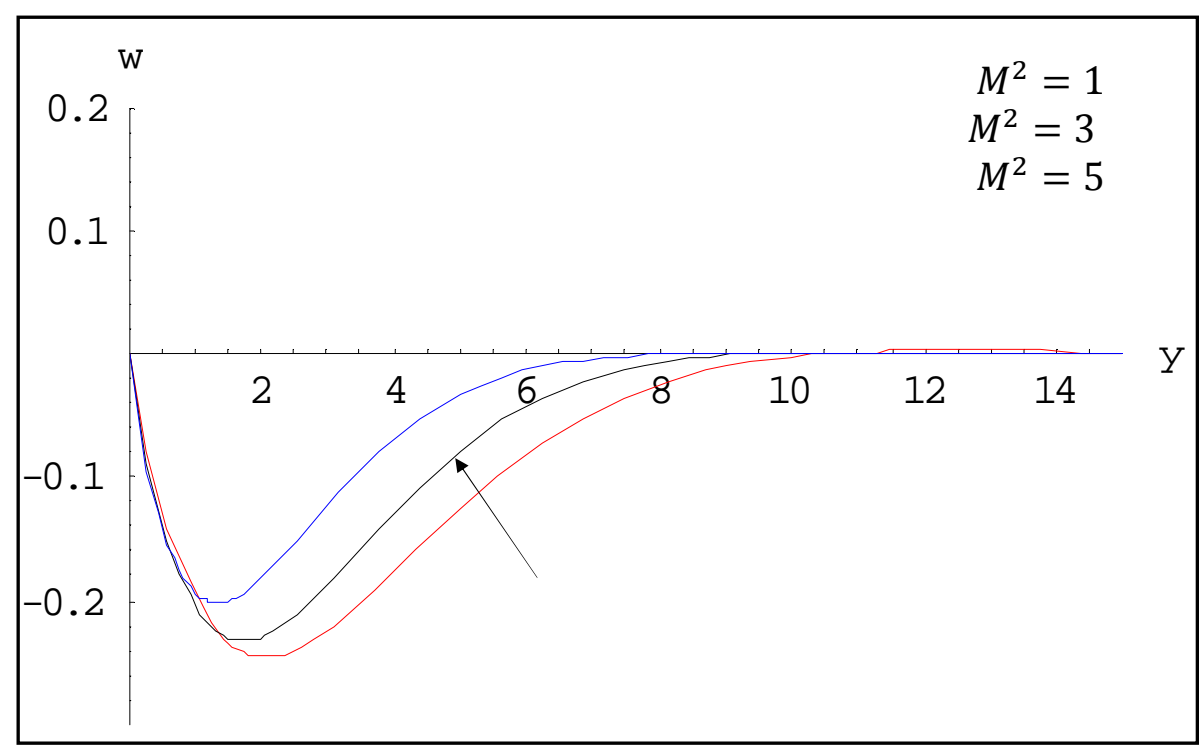

Figure 2: Effect of Hartmann number $\left(M^{2}\right)$ on secondary velocity profile when $m=1 ; \xi=1 ; \gamma=0.2 ; K^{2}=1 ; K_{p}=1$ 
P.Thirunavukarasu and S.Bhuvaneswari

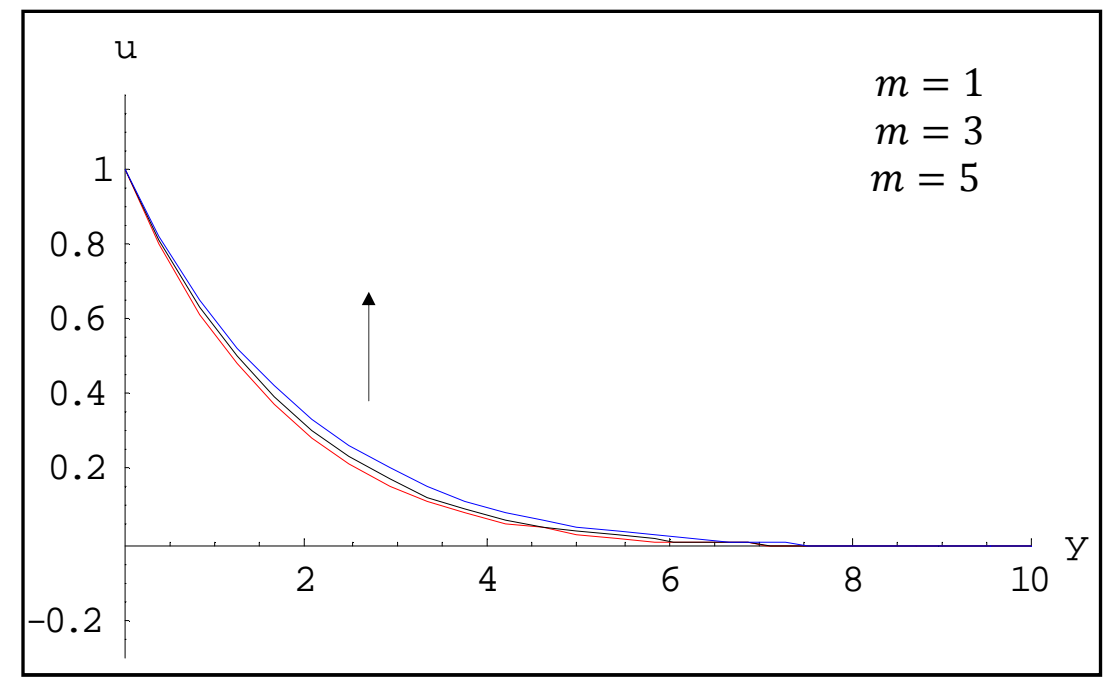

Figure 3: Effect of Hall parameter $(m)$ on primary velocity profile when $M^{2}=1 ; \xi=1 ; \gamma=0.2 ; K^{2}=1 ; K_{p}=1$

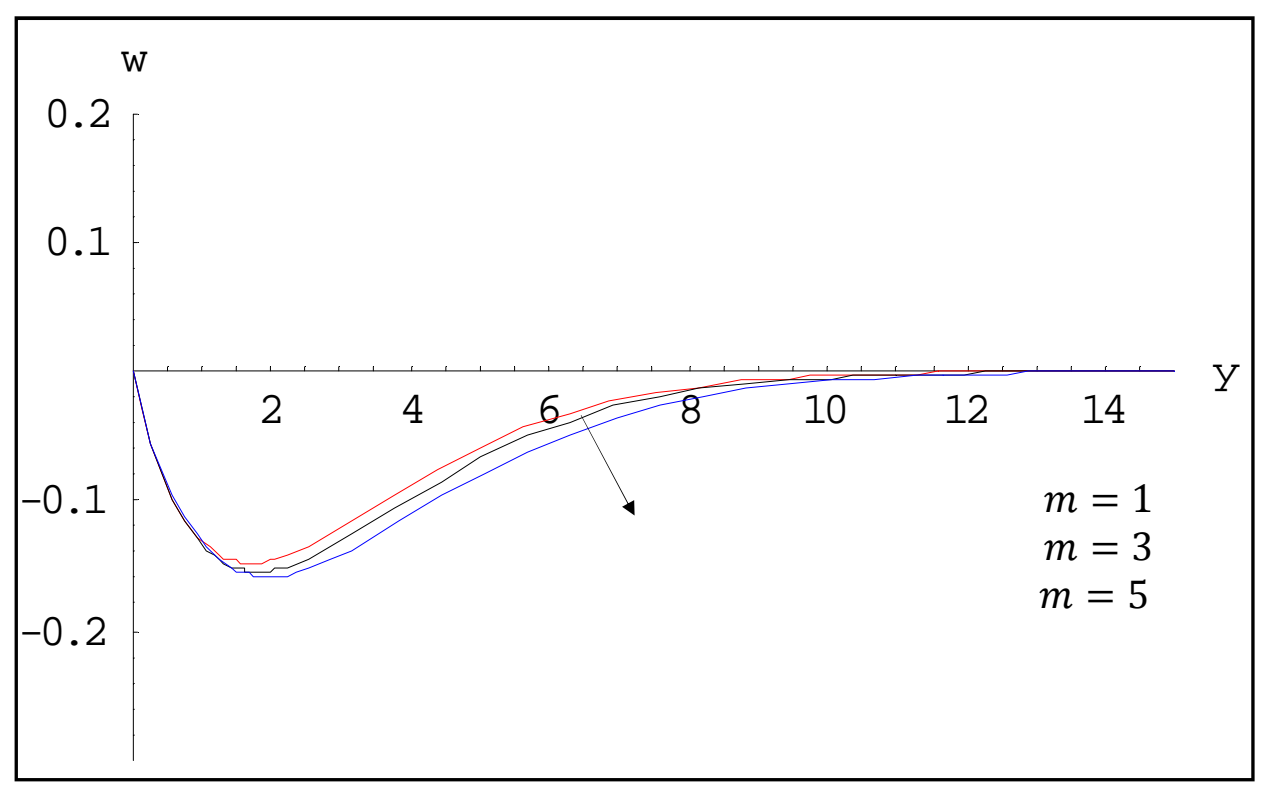

Figure 4: Effect of Hall parameter $(m)$ on secondary velocity profile when $M^{2}=1 ; \xi=1 ; \gamma=0.2 ; K^{2}=1 ; K_{p}=1$ 
Magnetohydrodynamic Casson Fluid Flow With Hall Effect and Rotation in a Porous Medium

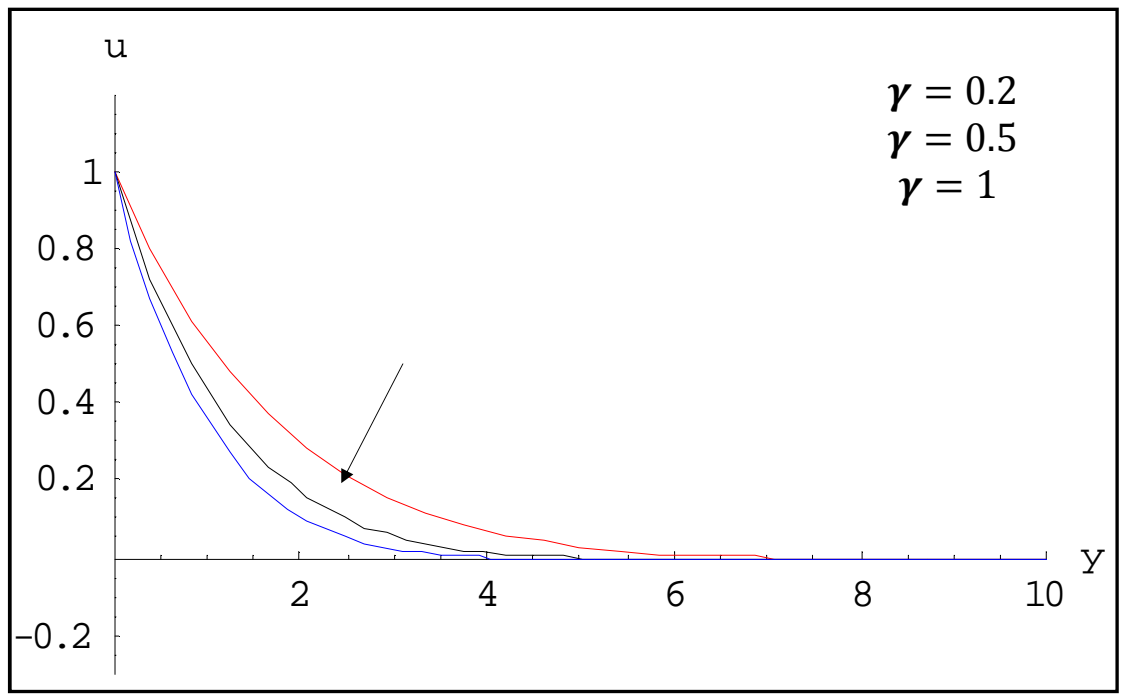

Figure 5: Effect of Casson fluid parameter $(\gamma)$ on primary velocity profile when $M^{2}=1 ; \xi=1 ; m=1 ; K^{2}=1 ; K_{p}=1$

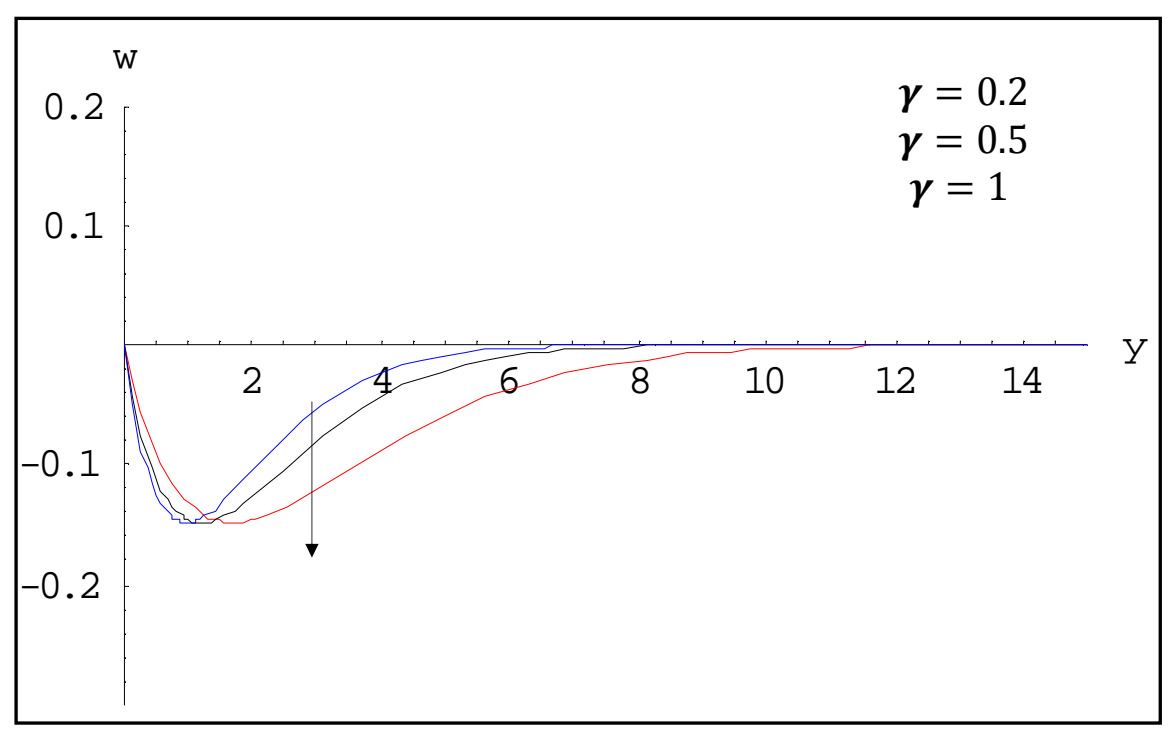

Figure 6: Effect of Casson fluid parameter $(\gamma)$ on secondary velocity profile when $M^{2}=1 ; \xi=1 ; m=1 ; K^{2}=1 ; K_{p}=1$ 
P.Thirunavukarasu and S.Bhuvaneswari

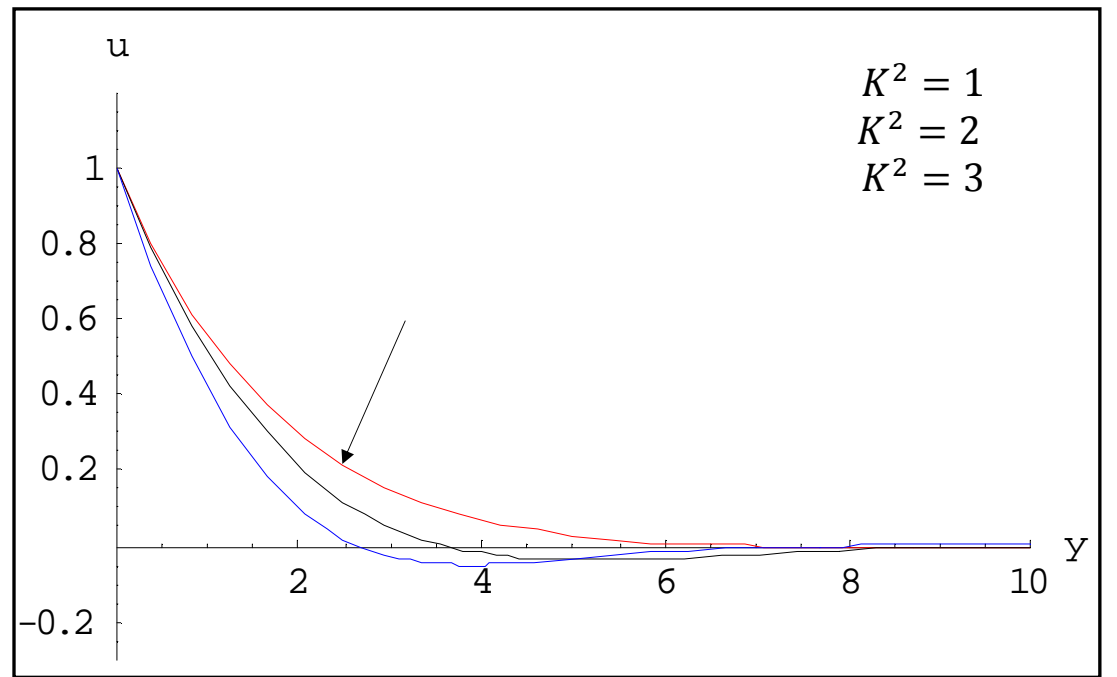

Figure 7: Effect of Rotation parameter $\left(K^{2}\right)$ on primary velocity profile when $m=1 ; \xi=1 ; \gamma=0.2 ; M^{2}=1 ; K_{p}=1$

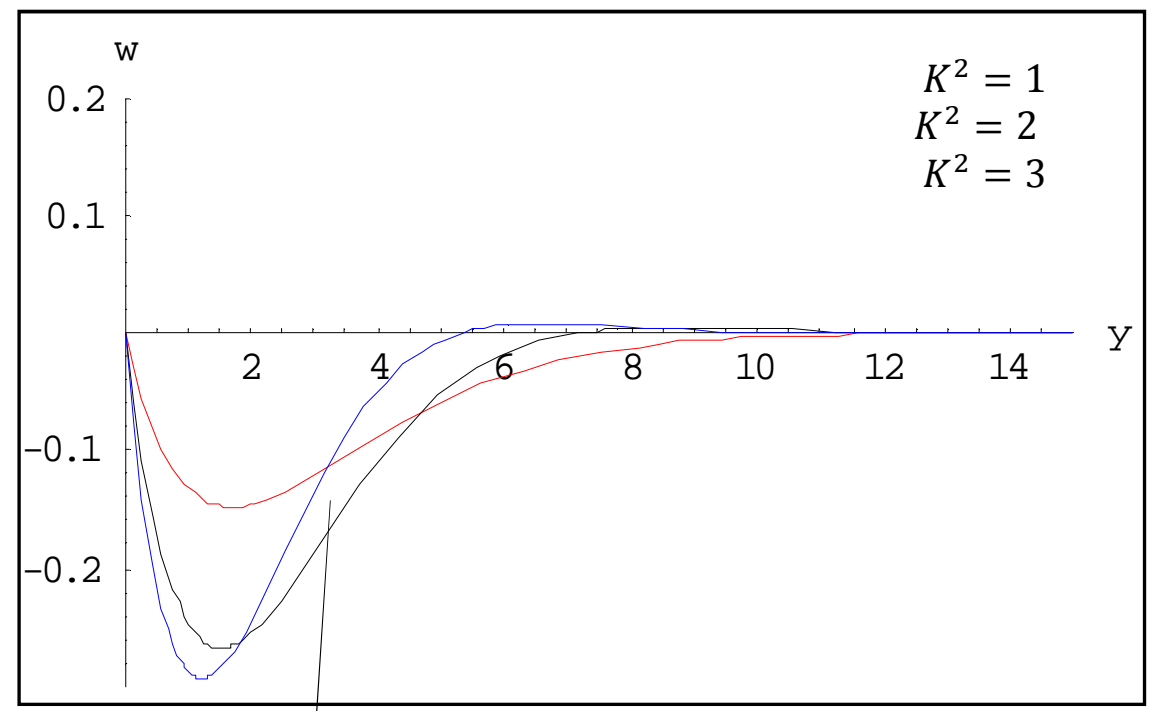

Figure 8: Effect of Rotation parameter $\left(K^{2}\right)$ on secondary velocity profile When $m=1 ; \xi=1 ; \gamma=0.2 ; M^{2}=1 ; K_{p}=1$ 
Magnetohydrodynamic Casson Fluid Flow With Hall Effect and Rotation in a Porous Medium

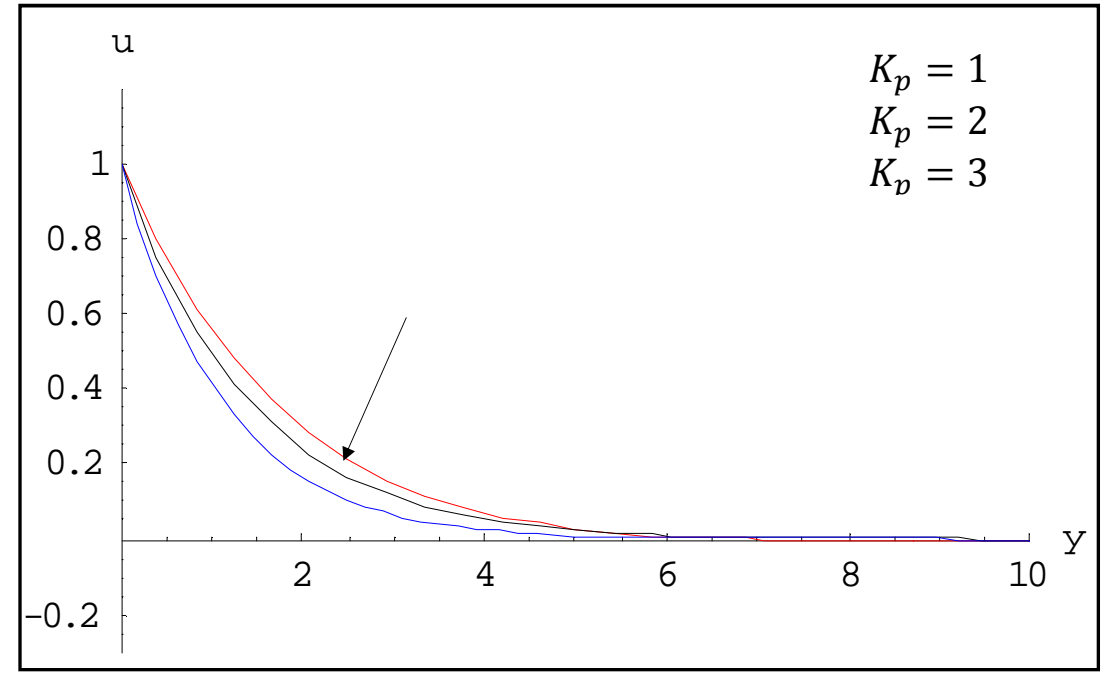

Figure 9: Effect of Porosity parameter $\left(K^{2}\right)$ on primary velocity profile when $m=1 ; \xi=1 ; \gamma=0.2 ; M^{2}=1 ; K^{2}=1$

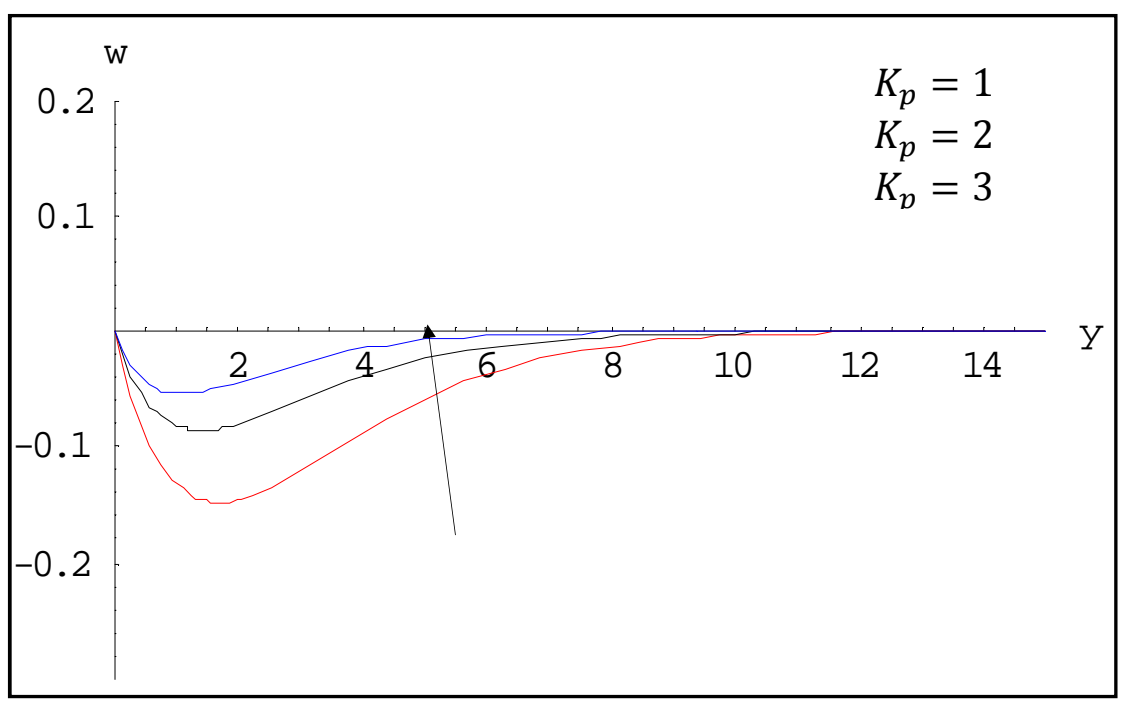

Figure 10: Effect of Porosity parameter $\left(K^{2}\right)$ on secondary velocity profile when $m=1 ; \xi=1 ; \gamma=0.2 ; M^{2}=1 ; K^{2}=1$ 
P.Thirunavukarasu and S.Bhuvaneswari

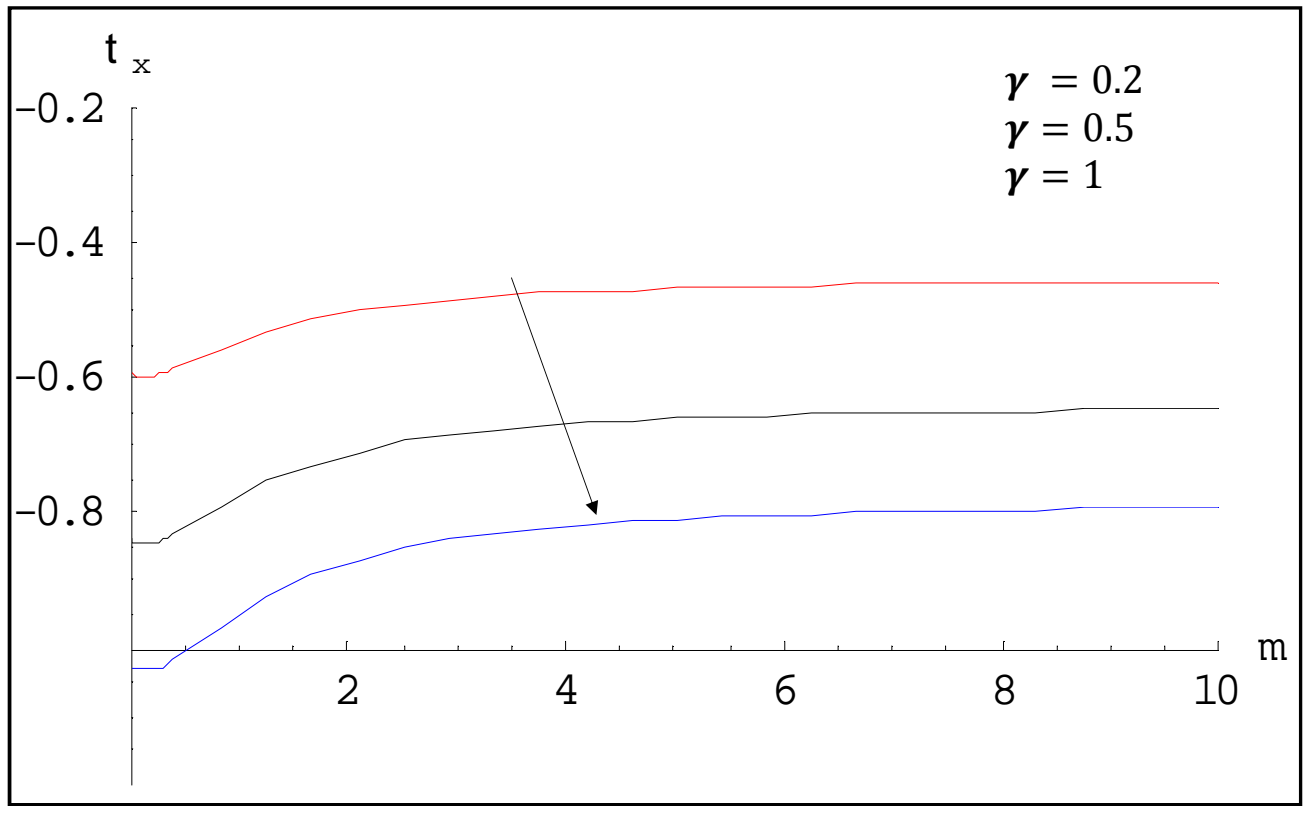

Figure 11: Shear stress $\tau_{x}$ for different $\gamma$ when $M^{2}=1 ; \xi=1 ; K^{2}=1 ; K_{p}=1$

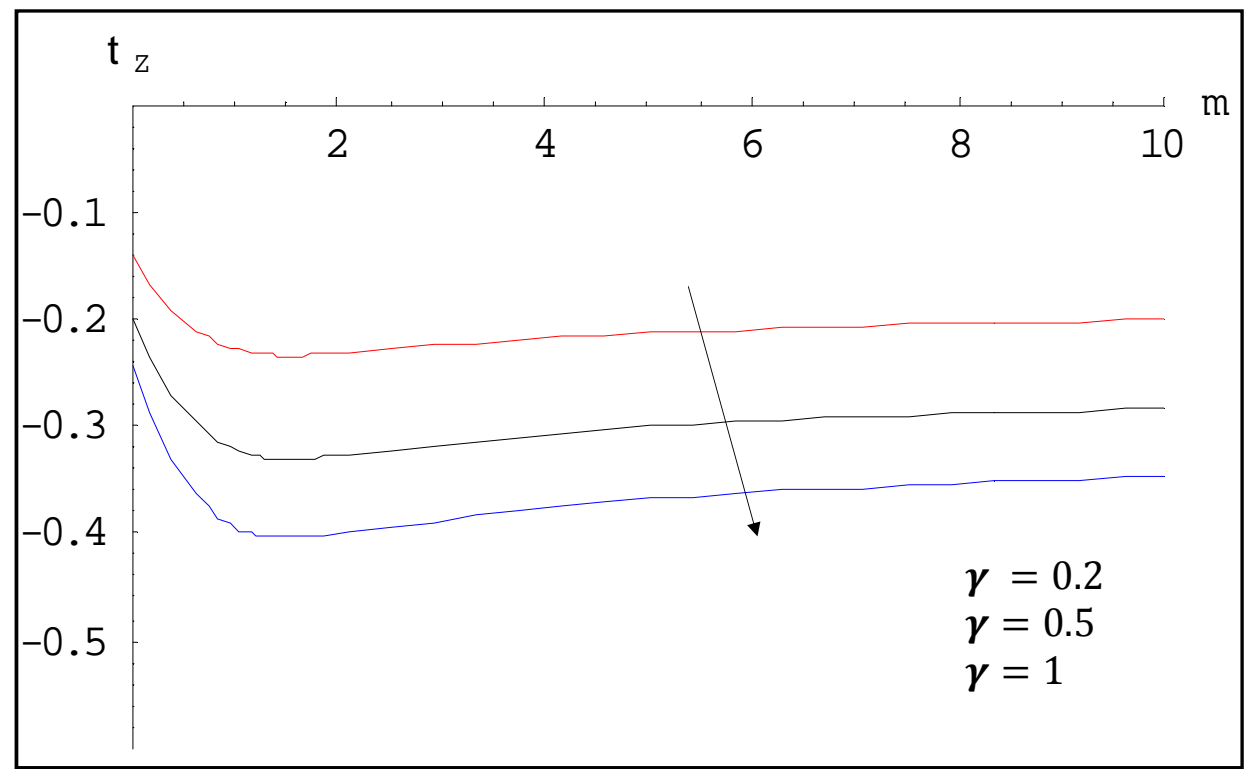

Figure 12: Shear stress $\tau_{z}$ for different $\gamma$ when $M^{2}=1 ; \xi=1 ; K^{2}=1 ; K_{p}=1$ 
Magnetohydrodynamic Casson Fluid Flow With Hall Effect and Rotation in a Porous Medium

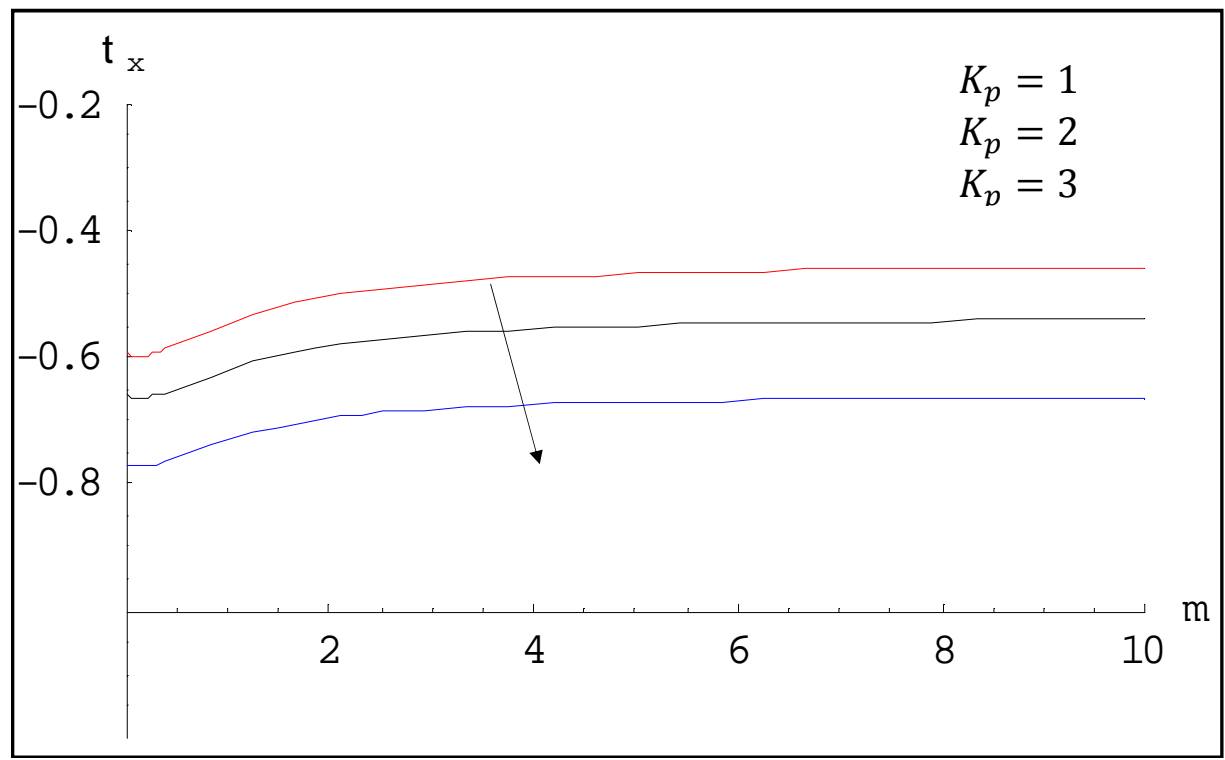

Figure 13: Shear stress $\tau_{x}$ for different $M^{2}$ when $\xi=1 ; \gamma=0.2 ; K^{2}=1 ; M^{2}=1$

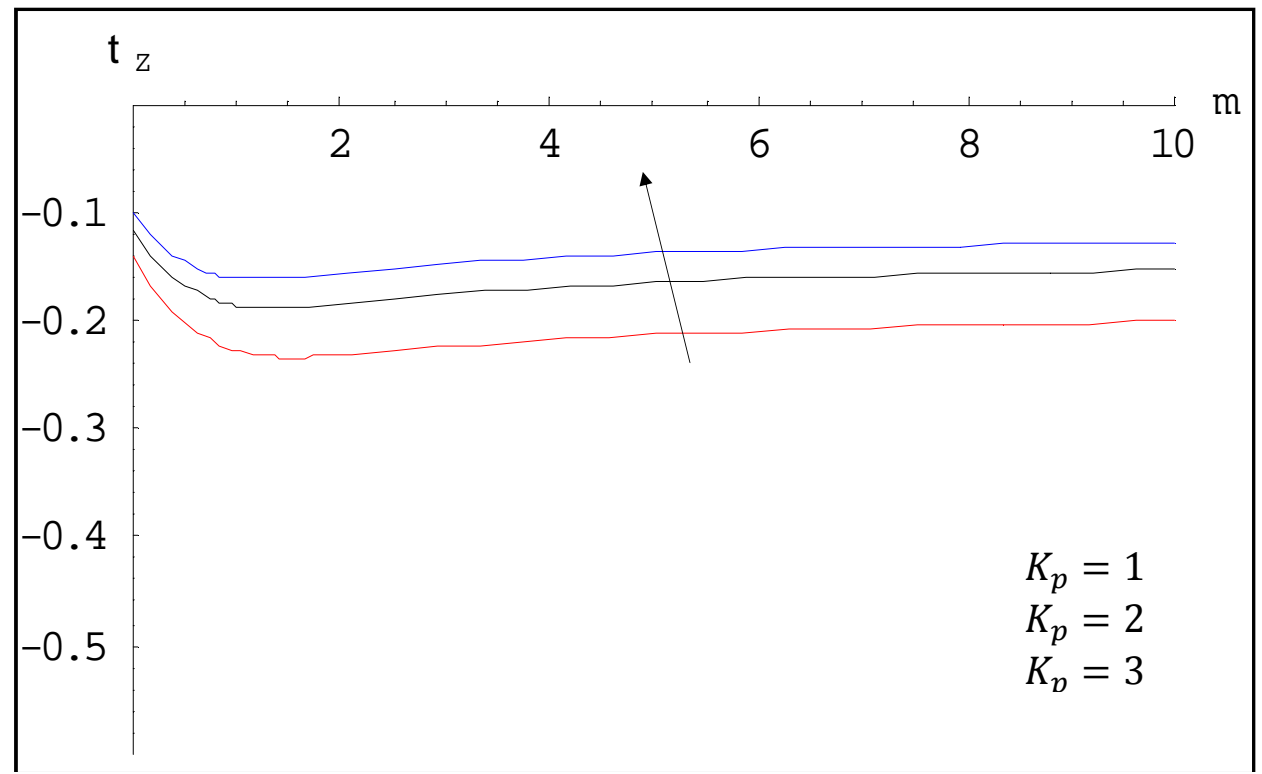

Figure 14: Shear stress $\tau_{z}$ for different $M^{2}$ when $\xi=1 ; \gamma=0.2 ; K^{2}=1 ; M^{2}=1$

\section{REFERENCES}


P.Thirunavukarasu and S.Bhuvaneswari

1. A.S.Gupta, Magnetohydrodynamic Ekman layer, Acta Mech., 13 (1972) 155-165.

2. L.Debnath, On unsteady magnetohydrodynamic boundary layers in a rotating fluid, ZAMM, 52 (1972) 523-527.

3. L.Debnath, Inertial Oscillationa and hydromagnetic multiple boundary layers In a rotating fluid, ZAMM, 55 (1975) 141-147.

4. S.K.Guchhait, S.Das and R.N.Jana, Combined effect of Hall current and Rotation on MHD mixed convection oscillating flow in a rotating vertical channel, International Journal of Computer Applications, 49(13) (2012) 1-11.

5. R.K.Dash, K.N.Mehta and G.Jayaraman, Casson fluid flow in a pipe filled with a homogeneous porous medium, International journal of Engineering Science, 34 (1996)1145-1156.

6. G.W.S.Blair, An equation for the flow of blood, plasma and serum through glass capillaries, Nature, 183 (1959) 613 -614.

7. S.Charm and G.Kurland, Viscometry of human blood for shear rates of 0100,000 sec $^{-1}$, Nature, 206 (1965) 617-618.

8. N.Casson, A flow equation for Pigment -oil suspension of the priniting in type. In: Mill, C.C., Ed., Rheology of disperse systems, Pergamon press, Oxford, 84-104, (1959). 